\title{
乌ु
}

\section{Collective Effects in Vortex Movements in Complex Plasmas}

\author{
Mierk Schwabe, ${ }^{1,2, *}$ Sergey Zhdanov, ${ }^{2}$ Christoph Räth, ${ }^{2}$ David B. Graves, ${ }^{1}$ Hubertus M. Thomas, ${ }^{2}$ and Gregor E. Morfill ${ }^{2}$ \\ ${ }^{1}$ Department of Chemical and Biomolecular Engineering, University of California, Berkeley, California 94720, USA \\ ${ }^{2}$ Max Planck Institute for Extraterrestrial Physics, P.O. Box 1312, Giessenbachstraße, 85741 Garching, Germany
}

(Received 12 August 2013; published 19 March 2014)

\begin{abstract}
We study the onset and characteristics of vortices in complex (dusty) plasmas using two-dimensional simulations in a setup modeled after the PK-3 Plus laboratory. A small number of microparticles initially self-arranges in a monolayer around the void. As additional particles are introduced, an extended system of vortices develops due to a nonzero curl of the plasma forces. We demonstrate a shear-thinning effect in the vortices. Velocity structure functions and the energy and enstrophy spectra show that vortex flow turbulence is present that is in essence of the "classical" Kolmogorov type.
\end{abstract}

DOI: 10.1103/PhysRevLett.112.115002

PACS numbers: 52.27.Lw, 52.35.Ra, 52.65.-y

Introduction.-Complex plasmas consist of micrometer-sized particles embedded in a low temperature plasma and are ideal model systems for nanofluids, phase transitions, transport processes, etc. [1]. They display many collective effects, for instance, vortices. These appear when gravity is compensated by thermophoresis [2-4] and in weightless systems [5-7] and can be induced externally, for instance, by a laser $[8,9]$, by a probe $[10,11]$, or by gas flow [12-15]. Vortices can accompany nucleation [16] and lead to shear flow instabilities [17]. The origin of self-excited vortices in complex plasmas is under discussion; they could be induced by the microparticle size dispersion $[3,18]$, charge gradients $[19,20]$, or a nonzero curl of the forces that the plasma exerts on the microparticles [21,22].

Vortices in complex plasmas are an ideal test bed for studying the onset of turbulence and collective effects on the microcanonical level [2]. The description and dynamics of a turbulent dusty plasma is an ongoing problem [23,24]. Turbulence can be induced by instabilities or waves in the dusty plasma [25-27]. In comparison with traditional experiments on turbulence (see, e.g., [28-30]), the particles that transmit the interaction can be visualized directly.

The opportunity to study turbulence in fluids with low Reynolds numbers (low- $\mathcal{R}$ turbulence) at the kinetic level is especially interesting. The emergence of low- $\mathcal{R}$ turbulence is important not only for complex plasmas $(\mathcal{R}=0.2-50[2,14,17])$, but also for many other physical, biophysical, and industrial applications as diverse as water flow through porous media $(\mathcal{R}=18-22$ [31], $\mathcal{R}=370$ [32]), viscoelastic liquid polymer flows $(\mathcal{R}<1[33,34])$, chaotic mixer $(\mathcal{R}<100$ [35]), self-propelled stochastic microdevices, and "bacterial" quasiturbulence $(\mathcal{R} \ll 1$ $[36,37])$, and the crossover from high- $\mathcal{R}$ to low- $\mathcal{R}$ turbulence $(\mathcal{R} \approx 20$ [38]). Low- $\mathcal{R}$ vortex movements are also important in fluids and the atmosphere, for instance, in insect flight [39]. Here, we use simulations of vortices in complex plasmas to investigate the onset of turbulence and collective effects.
Simulation particulars. -We use the simulation setup described in [40], which is a two-dimensional model of the central plane of the PK-3 Plus chamber [41]. It consists of a hybrid fluid-analytical description of the plasma by Kawamura et al. [42], to which a molecular-dynamics (MD) simulation of the microparticle dynamics is coupled. The MD simulation is based on the large-scale atomicmolecular massively parallel simulator (LAMMPS) [43], modified to take into account the plasma forces [44]. The microparticles interact via Yukawa pair potentials and are subject to random forces mimicking interactions with background atoms. The neutral gas exerts a friction force on the microparticles, the Epstein force [45]. The ion flux and ambipolar electric field from the plasma simulation cause the ion drag and confinement forces acting on the microparticles.

For the simulations presented here, we use argon at a pressure of $10 \mathrm{~Pa}$ as buffer gas. The microparticles have a diameter of $3.4 \mu \mathrm{m}$, a mass of $3.1 \times 10^{-14} \mathrm{~kg}$, and a charge of $-3481 e$. The friction constant with the background gas is $\gamma_{\mathrm{Ep}}=37 \mathrm{~s}^{-1}$. The electron temperature is $2.4 \mathrm{eV}$, the ion and neutral gas temperatures $300 \mathrm{~K}$. The mean electron and ion number densities are $3.4 \times 10^{14} \mathrm{~m}^{-3}$, with a maximum in the center of the simulation box (see [40]). We do not take into account the effect of the microparticles on the plasma. Unless otherwise mentioned, we used 7100 particles in the simulations runs.

Onset of vortices.-We mimic the experimental injection process by placing microparticles near the sheath edge. The ambipolar electric field pushes the microparticles into the plasma. Inside the plasma bulk, the ion drag force drives the microparticles around the void, where they arrange in a monolayer along the equilibrium line where the counteracting forces cancel. Figure 1 shows trajectories of particles inside this monolayer as progressively more particles are added (see Supplemental Material [46] for a movie). Adding single particles leads to transverse displacement and hopping of particles already inside the monolayer 


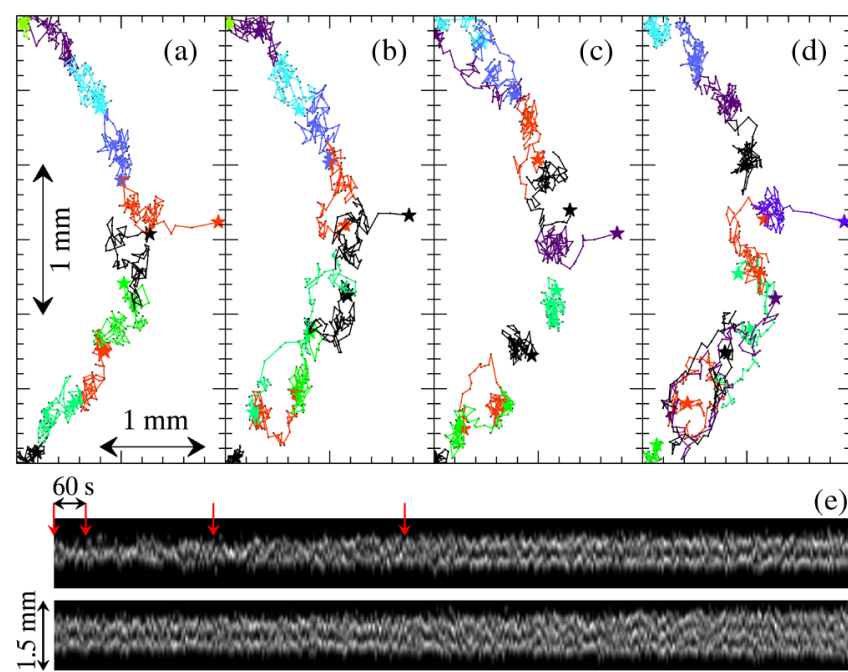

FIG. 1 (color online). (a)-(d) Trajectories that show the process of adding single particles to a monolayer. The individual trajectories are distinguished by color. Stars indicate the start points. (e) Space-time plot (periodgram) that demonstrates the development of the width of the central part $y=9.5-10.5 \mathrm{~mm}$ of the microparticle cloud with time (horizontal axis). For each time, the sum of the pixel intensities of an image of the particle positions is shown as a function of the $x$ position (vertical axis of the periodgram). Injections occur every minute. The arrows indicate the injections (a)-(d).

[Figs. 1(a)-1(c)]. After the 12th injection, two layers of particles form, and vortex motion ensues [Fig. 1(d)]. The space-time plot (periodgram) [Fig. 1(e)] demonstrates the growth of the cloud width with additional injections.

Figure 2 shows the vector plot of the combined plasma forces acting on the particles, $\mathbf{F}=\mathbf{F}_{i}+\mathbf{F}_{e}$, namely, the sum of the ion drag force $\mathbf{F}_{i}$ and the ambipolar electric force $\mathbf{F}_{e}$ [40]. On the outside of the equilibrium line, $\mathbf{F}_{e}$ dominates, whereas on the inside, $\mathbf{F}_{i}$ dominates, keeping the central "void" particle free. The cause of the finite width

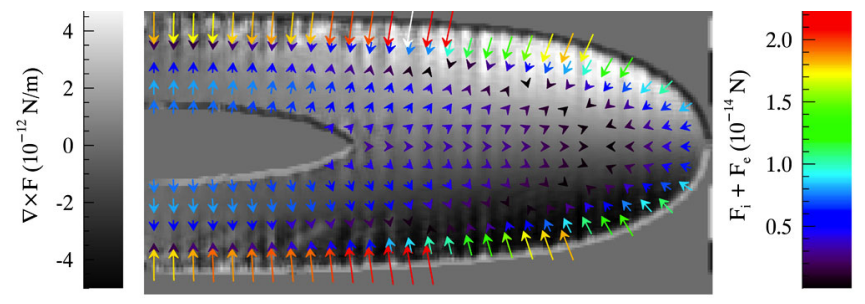

FIG. 2 (color online). The sum of the ion drag $F_{i}$ and electric force $F_{e}$ acting on the particles (vectors, color online, right color map) overlaid a map of the curl of the force field (grayscale, left color map). The equilibrium line is clearly visible in the vector field. The edge of the cloud in the curl map is enhanced, which is an artifact of the calculation. (See Supplemental Material [46] for a map of the absolute value of the curl of the force field.) Both curl maps use a threshold of $\pm 5 \times 10^{-12} \mathrm{~N} / \mathrm{m}$ to enhance the contrast. of the microparticle cloud is the mutual interparticle repulsion.

The grayscale background of Fig. 2 maps the nonzero component of the curl of the plasma force field, $\nabla \times \mathbf{F}$ [47]. The magnitude of the curl of the vector field is largest near the edges of the microparticle cloud, whereas it nearly vanishes on the inside of the equilibrium line and in the midplane of the simulation box. This qualitatively confirms the basic result of Akdim and Goedheer [21]: the vortices can be caused by a nonvanishing curl of the plasma force, which our estimates show to most probably originate from the cross product of gradients of electron temperature and plasma density (see also [48]).

Fully developed system of vortices.-Once several layers of microparticles are present, the vortices are fully developed. Figure 3 visualizes the movement of microparticles inside a cloud with two vortices. The particles move towards the simulation box center along the outside of the cloud and back towards the cloud edge in the central plane, as in the experiments in the PK-3 Plus laboratory on board the International Space Station [49].

The particles move the fastest at the edge of the vortices. We calculate the enstrophy density following [17]: First, we determine the $n n$ closest neighbors of every particle $i$ ( $i=1, \ldots, N$, where $N$ is the total number of particles), and find the vectors to their positions $\mathbf{r}_{i j}=\mathbf{r}_{j}-\mathbf{r}_{i}$ and the relative velocities $\mathbf{v}_{i j}=\mathbf{v}_{j}-\mathbf{v}_{i}$ for every neighbor

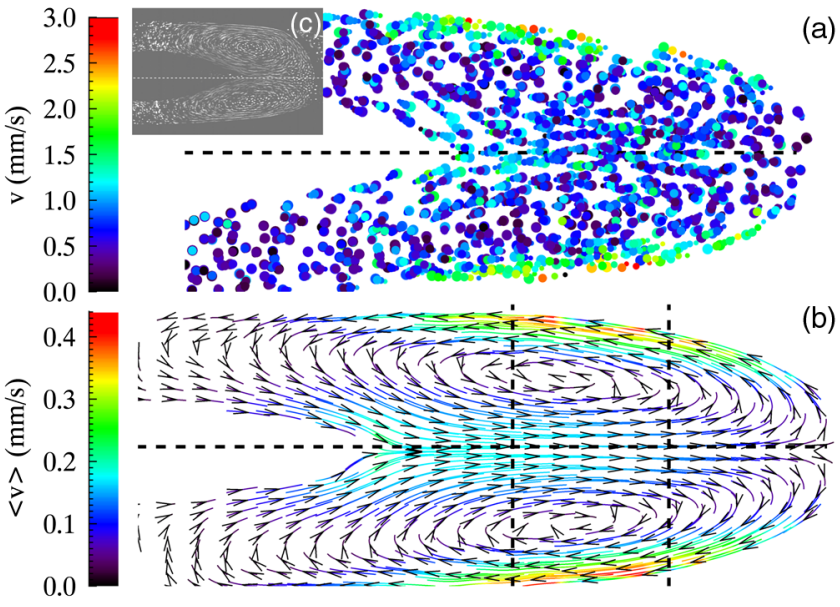

FIG. 3 (color online). Simulated (a), (b) and experimental vortices (c). The horizontal dashed lines mark the chamber midplane and simulation box center. The field of view is (a), (b) $23 \times 10 \mathrm{~mm}^{2}$, and (c) $30 \times 20 \mathrm{~mm}^{2}$. (a) Positions of $1 / 10$ of all particles in the simulation. The dots indicate four particle positions $0.2 \mathrm{~s}$ apart for each particle and grow with time. The color of the dots indicates the total particle velocity, which is shown with a threshold of $3 \mathrm{~mm} / \mathrm{s}$. (b) Streamlines and arrows indicate the mean velocity field of the simulated vortices. The vertical dashed lines indicate the position of the sliding window used for Fig. 4. (c) Overlay of 60 pictures (30 s) taken with the PK-3 Plus laboratory, mirrored horizontally. Courtesy of the PK-3 Plus team [41]. 
$j=1, \ldots, n n$. The vorticity $\omega_{i}$ is a function of the projections $c_{i j}$ of the relative velocity onto the vectors orthogonal to $\mathbf{r}_{i j}$ :

$$
\omega_{i}=\mathbf{e}_{z} \cdot(\operatorname{curl} \mathbf{v})_{i}=\frac{1}{n n} \sum_{j=1}^{n n} c_{i j},
$$

where, $\mathbf{e}_{z}=\mathbf{e}_{x} \times \mathbf{e}_{y}$ is the unit vector in direction perpendicular to the plane of the simulation, and $c_{i j}=\left(\mathbf{e}_{z} \times \mathbf{r}_{i j}\right) \cdot \mathbf{v}_{i j} / r_{i j}^{2}$, where the indices $i$ and $j$ are as introduced above. The number of neighbors used in the calculation is $n n=16$. Finally, we calculate the enstrophy density $\Omega_{k l}$ in every grid cell $(k, l)$ from the average squared vorticity

$$
\Omega_{k l}=\left(\frac{1}{n} \sum_{i=0}^{n} \omega_{i}\right)^{2}
$$

where $n$ is the number of particles in the cell.

The enstrophy density is concentrated at the edges of the microparticle cloud (see Supplemental Material [46]), where the microparticles move the fastest. As there are considerable velocity differences across the cloud, we shall next see whether the vortices produce any shear stress.

Shear thinning.-We follow [50] to calculate the shear stress $\sigma_{x y}$, i.e., the off diagonal elements of the pressure tensor, that is buildup from the contributions of each particle's neighbors, neglecting the kinetic component. For a two-dimensional system in polar coordinates, $\sigma_{x y}$ is given by

$$
\sigma_{x y}=-\frac{1}{2} n^{2} \int_{0}^{r_{\max }} d r r^{2} \frac{d V}{d r} \int_{0}^{2 \pi} d \varphi \cos \varphi \sin \varphi g(r, \varphi),
$$

where $n$ is the number density of the microparticles, $V$ is the Yukawa potential, and $g(r, \phi)$ is the radial pair distribution function. The insets (a) and (b) in Fig. 4 show the pair correlation functions at the upper and lower edges of the cloud. The elliptical distortion of $g(r, \phi)$ shows strong shear stress.

The main part of Fig. 4 shows the shear rate $\gamma=\left(\partial v_{x} / \partial y\right)+\left(\partial v_{y} / \partial x\right)$, calculated from the velocity maps, and the shear stress $\sigma_{x y}$ as a function of height above the lower sheath edge in a sliding window of size $5 \times 2 \mathrm{~mm}^{2}$ at a horizontal distance of $12 \mathrm{~mm}$ from the plasma center (see Fig. 3). The vertical center of the simulation box is at a height of $10 \mathrm{~mm}$. Both the shear rate and the shear stress vanish in the middle, where all particles are moving towards the edge of the cloud. Their absolute values are largest close to the area where the opposite fluxes induced by the vortices meet. Numerically, the values are close to those found in experiments by Nosenko et al. [50]. We also observe shear thinning, as can

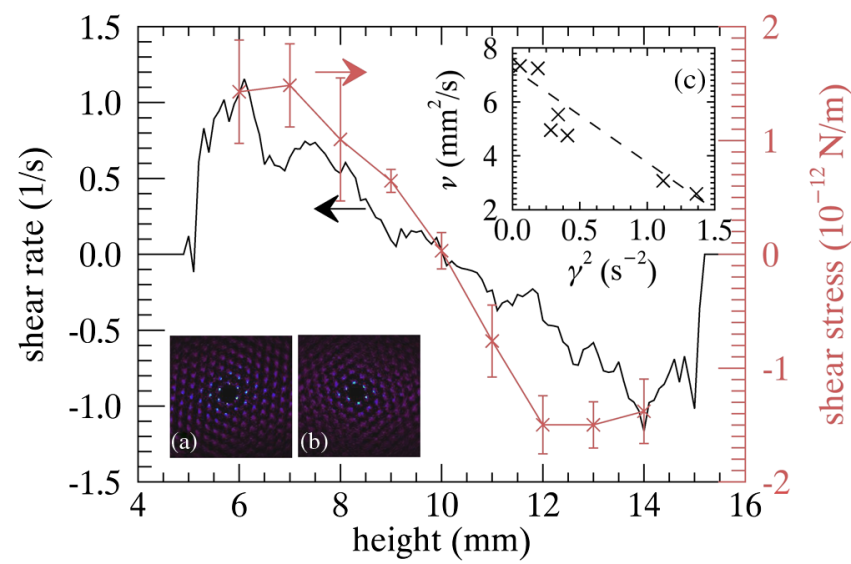

FIG. 4 (color online). Shear rate and shear stress as a function of height above the lower sheath edge. Insets: radial pair correlation functions at the lower (a) and upper (b) edges of the cloud, (c) viscosity $\nu$ as a function of squared shear stress $\gamma^{2}$, demonstrating shear thinning. The dashed line is a guide to the eye.

be seen in Fig. 4(c) [51]: The viscosity $\nu=\sigma_{x y} /(\rho \gamma)$, with $\rho$ being the areal mass density, decreases as a function of squared shear stress.

Flow velocity.-The parameters of the simulated vortex flow fit real experiments performed with complex plasmas. For instance, using a mean viscosity of $\nu=5 \mathrm{~mm}^{2} / \mathrm{s}$, a flow velocity of $v=2 \mathrm{~mm} / \mathrm{s}$, and a typical size of the vortices of $L=10 \mathrm{~mm}$ (see Figs. 3 and 4), we obtain a Reynolds number $\mathcal{R}=v L / \nu \sim 4$, which is well in-line with observations $[2,14,17]$. We do find evidence that turbulence is present in the system of vortices at such a low $\mathcal{R}$ value.

Turbulence.-The origin of the turbulent pulsations in our simulations is twofold: the external normally distributed random forces and the mutual interparticle interactions leading to randomization. Using the average viscosity $\nu=5 \mathrm{~mm}^{2} / \mathrm{s}$ and the mean enstrophy density $\Omega=0.4 \mathrm{~s}^{-2}$, we get an energy dissipation rate per unit mass [52] of the order of $\epsilon=2 \nu \Omega=4 \mathrm{~mm}^{2} / \mathrm{s}^{3}$. This results in a Kolmogorov length scale of $L_{K}=\left(\nu^{3} / \epsilon\right)^{1 / 4}=2.4 \mathrm{~mm}$, which is an intermediate scale between the size of the simulation box $\left(20 \times 45 \mathrm{~mm}^{2}\right)$ and the interparticle distance $(0.15 \mathrm{~mm})$.

The longitudinal structure functions of order $p, S_{p}(r)$, are given by

$$
S_{p}(r)=\left\langle\left(\Delta u_{r}\right)^{p}\right\rangle=\left\langle[u(x+r)-u(x)]^{p}\right\rangle,
$$

where $u(x)$ is the velocity component parallel to the relative displacement $r$. We use the absolute value of the velocity difference in the calculations [28]. For fully developed turbulence, the structure functions show a power law scaling [52], $S_{p} \propto r^{\zeta_{p}}$. Kolmogorov [53] predicted the exponents to be given by $\zeta_{p}=p / 3$. Figure 5 shows the horizontal velocity structure functions measured in the 


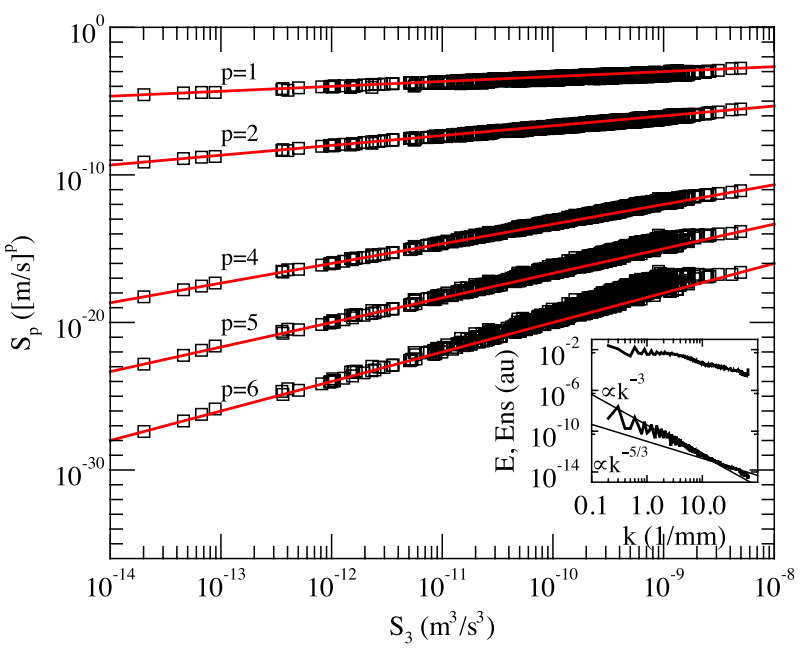

FIG. 5 (color online). Squares: first $(p=1)$ through sixth $(p=6)$ order longitudinal velocity structure functions $S_{p}$ as a function of the third order structure function $S_{3}$. The lines are those predicted by classical Kolmogorov theory $S_{p} \propto r^{\zeta_{p}}$, with $\zeta_{p}=p / 3$. Inset: energy (bottom) and enstrophy density spectrum (top). The slopes of the lines overplotted on the energy spectrum are -3 and $-5 / 3$.

region to the right of the void. Following Lewis and Swinney [28], we use extended self-similarity [54], i.e., we plot $S_{p}$ vs $S_{3}$ on a log-log plot and use the fact that $\zeta_{3}=1$ according to Kolmogorov's theory. The slopes of the plots are then given by $\zeta_{p} / \zeta_{3}=\zeta_{p}$. Figure 5 shows that the slopes are very close to those predicted by Kolmogorov (as the overplotted solid lines in the figure indicate).

The inset in Fig. 5 shows the energy and enstrophy density spectra. The energy spectrum is calculated from the sum of the squares of the Fourier transformed velocity maps (see Fig. 3), the enstrophy spectrum from the enstrophy map (see Supplemental Material [46]). The two lines that are overplotted on the energy spectrum have the slopes -3 and $-5 / 3$, as expected: The $\propto k^{-5 / 3}$ dependence is typical inside the inertial range, and the crossover to $\propto k^{-3}$ is due to friction [52].

Summary.-We numerically studied two-dimensional vortices in a complex plasma. We showed how these vortices develop from a monolayer around the void, and that a nonzero curl of the combined ion drag and electric forces causes the vortex movement of the microparticles. We then investigated the properties of a fully developed system of vortices. In particular, we showed that turbulence is present in the flow induced by the vortices and demonstrated that the velocity structure functions scale very close to the predictions by Kolmogorov theory. These results show that it should be possible to use common experimental situations like vortices in complex plasmas to study turbulence on the level of individual particles.

We acknowledge support by a Marie Curie International Outgoing Fellowship within the 7th European Community
Framework Programme, from the European Research Council under the European Union's Seventh Framework Programme (Program No. FP7/2007-2013)/ERC Grant Agreement No. 267499, and by the US Department of Energy, Office of Fusion Science Plasma Science Center. The corresponding experiments on the International Space Station were funded by DLR/BMWi under the Contracts No. FKZs 50 WM 0203 and No. 50 WM 1203.

*mierk@berkeley.edu

[1] G. E. Morfill and A. V. Ivlev, Rev. Mod. Phys. 81, 1353 (2009).

[2] G. E. Morfill, M. Rubin-Zuzic, H. Rothermel, A. V. Ivlev, B. A. Klumov, H. M. Thomas, U. Konopka, and V. Steinberg, Phys. Rev. Lett. 92, 175004 (2004).

[3] M. Rubin-Zuzic, H. Thomas, S. Zhdanov, and G. E. Morfill, New J. Phys. 9, 39 (2007).

[4] M. Schwabe, M. Rubin-Zuzic, S. Zhdanov, A. V. Ivlev, H. M. Thomas, and G. E. Morfill, Phys. Rev. Lett. 102, 255005 (2009).

[5] G. E. Morfill, H. M. Thomas, U. Konopka, H. Rothermel, M. Zuzic, A. Ivlev, and J. Goree, Phys. Rev. Lett. 83, 1598 (1999).

[6] V. E. Fortov, O. S. Vaulina, O. F. Petrov, V. I. Molotkov, A. V. Chernyshev, A. M. Lipaev, G. Morfill, H. Thomas et al., JETP Lett. 96, 704 (2003).

[7] A. P. Nefedov, G. E. Morfill, V. E. Fortov, H. M. Thomas, H. Rothermel, T. Hagl, A. V. Ivlev, M. Zuzic et al., New J. Phys. 5, 33 (2003).

[8] M. Klindworth, A. Melzer, A. Piel, and V. A. Schweigert, Phys. Rev. B 61, 8404 (2000).

[9] T. Miksch and A. Melzer, Phys. Rev. E 75, 016404 (2007).

[10] D. A. Law, W. H. Steel, B. M. Annaratone, and J. E. Allen, Phys. Rev. Lett. 80, 4189 (1998).

[11] G. Uchida, S. Iizuka, T. Kamimura, and N. Sato, Phys. Plasmas 16, 053707 (2009).

[12] V. I. Vladimirov, L. V. Deputatova, A. P. Nefedov, V. E. Fortov, V. A. Rykov, and A. V. Khudyakov, J. Exp. Theor. Phys. 93, 313 (2001).

[13] S. Mitic, R. Sütterlin, A. V. Ivlev, H. Höfner, M. H. Thoma, S. Zhdanov, and G. E. Morfill, Phys. Rev. Lett. 101, 235001 (2008).

[14] M. Schwabe, L.-J. Hou, S. Zhdanov, A. V. Ivlev, H. M. Thomas, and G. E. Morfill, New J. Phys. 13, 083034 (2011).

[15] M. A. Fink, S. K. Zhdanov, M. Schwabe, M. H. Thoma, H. Höfner, H. M. Thomas, and G. E. Morfill, Europhys. Lett. 102, 45001 (2013).

[16] V. Nosenko, S. Zhdanov, and G. Morfill, Phys. Rev. Lett. 99, 025002 (2007).

[17] R. Heidemann, S. Zhdanov, K. R. Sütterlin, H. M. Thomas, and G. E. Morfill, Europhys. Lett. 96, 15001 (2011).

[18] T. Yokota and K. Honda, J. Quant. Spectrosc. Radiat. Transfer 56, 761 (1996).

[19] O. S. Vaulina, A. P. Nefedov, O. F. Petrov, and V. E. Fortov, J. Exp. Theor. Phys. 91, 1147 (2000).

[20] O. S. Vaulina, A. A. Samarian, O. F. Petrov, B. W. James, and V. E. Fortov, New J. Phys. 5, 82 (2003). 
[21] M. R. Akdim and W. J. Goedheer, Phys. Rev. E 67, 056405 (2003).

[22] W. J. Goedheer and M. R. Akdim, Phys. Rev. E 68, 045401 (R) (2003).

[23] K. Rypdal, B. Kozelov, S. Ratynskaia, B. Klumov, C. Knapek, and M. Rypdal, New J. Phys. 10, 093018 (2008).

[24] A. Yu. Pigarov, S. I. Krasheninnikov, T. K. Soboleva, and T. D. Rognlien, Phys. Plasmas 12, 122508 (2005).

[25] P. K. Shukla, R. Bharuthram, and R. Schlickeiser, Phys. Plasmas 11, 1732 (2004).

[26] J. Pramanik, B. Veeresha, G. Prasad, and P. Kaw, Phys. Lett. A 312, 84 (2003).

[27] Y.-Y. Tsai, M.-C. Chang, and L. I, Phys. Rev. E 86, 045402 (2012).

[28] G. S. Lewis and H. L. Swinney, Phys. Rev. E 59, 5457 (1999).

[29] A. Arnèodo, R. Benzi, J. Berg, L. Biferale, E. Bodenschatz, A. Busse, E. Calzavarini, B. Castaing et al., Phys. Rev. Lett. 100, 254504 (2008).

[30] R. Monchaux, New J. Phys. 14, 095013 (2012).

[31] J. W. Fox, Proc. Phys. Soc. London Sect. B 62, 829 (1949).

[32] N. A. Horton and D. Pokrajac, Phys. Fluids 21, 045104 (2009).

[33] Ch. Goddard, O. Hess, and S. Hess, Phys. Rev. E 81, 036310 (2010); L. Pan, A. Morozov, C. Wagner, and P. E. Arratia, Phys. Rev. Lett. 110, 174502 (2013).

[34] A. Groisman and V. Steinberg, Nature (London) 405, 53 (2000).

[35] A. D. Stroock, S. K.W. Dertinger, A. Ajdari, I. Mezić, H. A. Stone, and G. M. Whitesides, Science 295, 647 (2002).

[36] L. H. Cisneros, R. Cortez, Ch. Dombrowski, R. E. Goldstein, and J. O. Kessler, Exp. Fluids 43, 737 (2007); Ch. W. Wolgemuth, Biophys. J. 95, 1564 (2008); A. M. Ardekani and R. Stocker, Phys. Rev. Lett. 105, 084502 (2010).

[37] R. Golestanian and A. Ajdari, J. Phys. Condens. Matter 21, 204104 (2009); L. E. Becker, S. A. Koehler, and H. A. Stone, J. Fluid Mech. 490, 15 (2003); R. Golestanian, Eur. Phys. J. E 25, 1 (2008); P. Tierno, R. Golestanian, I. Pagonabarraga, and F. Sague, J. Phys. Chem. B 112, 16525 (2008).

[38] D. Lohse, Phys. Rev. Lett. 73, 3223 (1994).

[39] Z. J. Wang, Phys. Rev. Lett. 85, 2216 (2000).
[40] M. Schwabe and D. B. Graves, Phys. Rev. E 88, 023101 (2013).

[41] H. M. Thomas, G.E. Morfill, V.E. Fortov, A. V. Ivlev, V. I. Molotkov, A. M. Lipaev, T. Hagl, H. Rothermel et al., New J. Phys. 10, 033036 (2008).

[42] E. Kawamura, D. B. Graves, and M. A. Lieberman, Plasma Sources Sci. Technol. 20, 035009 (2011).

[43] S. Plimpton, J. Comput. Phys. 117, 1 (1995).

[44] The "plasma forces" in our simulation are represented by the sum of the electric field force and the ion drag force. The analytical expressions to compute them are given in Ref. [40].

[45] P. Epstein, Phys. Rev. 23, 710 (1924).

[46] See Supplemental Material at http://link.aps.org/ supplemental/10.1103/PhysRevLett.112.115002 for a movie showing the addition of individual particles to a monolayer, for a map of the absolute value of the curl, and for a figure showing the distribution of enstrophy density.

[47] The enhancement of the borders of the cloud is an artifact of the calculation: the implemented algorithm overestimates the curl value at the edge of the cloud.

[48] As is easy to check, this also readily follows from a simplified rotation balance: $|\nabla \times \mathbf{v}| \sim\left(m \gamma_{\mathrm{eff}}\right)^{-1}|\nabla \times \mathbf{F}|$, where $\gamma_{\text {eff }}=\gamma_{\mathrm{Ep}}+\nu k_{\text {eff }}^{2}$ with $k_{\text {eff }} \sim 2 \pi / L_{K}$.

[49] Note that in experiments, more complicated situations exist as well, for instance, a reversal of the direction of rotation or a system with several vortices, which we have not observed in simulations so far.

[50] V. Nosenko, A. V. Ivlev, and G. E. Morfill, Phys. Rev. E 87, 043115 (2013).

[51] Values with $\gamma^{2}<0.05 \mathrm{~s}^{-2}$ were omitted in the plot, as the viscosity cannot be calculated by the above formula for $\gamma^{2}$ that are too small [50].

[52] U. Frisch, Turbulence : The Legacy of A. N. Kolmogorov (Cambridge University Press, Cambridge, 1995).

[53] A. N. Kolmogorov, Dokl. Akad. Nauk SSSR 30, 301 (1941) [Proc. R. Soc. London A 434, 9 (1991)]; Dokl. Akad. Nauk SSSR 32, 19 (1941) [Proc. R. Soc. London A 434, 15 (1991)].

[54] R. Benzi, S. Ciliberto, R. Tripiccione, C. Baudet, F. Massaioli, and S. Succi, Phys. Rev. E 48, R29 (1993). 